Vietnam Journal of Mechanics, VAST, Vol.41, No. 4 (2019), pp. 363 -371

DOI: https://doi.org/10.15625/0866-7136/14621

\title{
DISPERSION EQUATION OF RAYLEIGH WAVES IN TRANSVERSELY ISOTROPIC NONLOCAL PIEZOELASTIC SOLIDS HALF-SPACE
}

\author{
Do Xuan Tung \\ Hanoi Architectural University, Vietnam \\ E-mail: tungdx2783@gmail.com
}

Received: 12 October 2019 / Published online: 23 December 2019

\begin{abstract}
This study is devoted to investigate the propagation of Rayleigh-type waves in transversely isotropic nonlocal piezoelastic half-space. When the stress-free boundary is maintained at charge-free condition, the dispersion equation for the propagation of Rayleigh waves at the free surface of transversely isotropic piezoelastic solids has been obtained. Based on the obtained dispersion equation, the effect of the nonlocality on the speed of Rayleigh wave is numerically considered. The dependence of velocities of plane waves in transversely isotropic nonlocal piezoelastic medium on the direction of propagation as well as non-dimensional frequency parameter has been also illustrated.
\end{abstract}

Keywords: dispersion equation, nonlocal, piezoelastic.

\section{INTRODUCTION}

In recent years piezoelectric materials has drawn much attention towards application in surface acoustic wave (SAW) micro sensors, energy harvesting structure, health monitoring systems, transducers and actuators, etc. Both theoretical and experimental studies on wave propagation in piezoelectric materials have attracted the attention of scientists and engineers during last two decades. The survey of literature can be found in many related texts and books [1,2]. We mention only a few such as: Zinchuk and Podlipenets [3] obtained dispersion equations for acousto-electric Rayleigh wave in a periodic layer piezoelectric half-space in a study for the $6 \mathrm{~mm}$ crystal class. Wave propagation in porous piezoelectric material (PPM), having crystal symmetry $6 \mathrm{~mm}$, is studied analytically by Vashishth et al. [4]. Sharma et al. [1] investigated the propagation of Rayleigh waves in a homogeneous, transversely isotropic, piezothermoelastic half-space subjected to stress free, electrically shorted/charge-free and thermally insulated/isothermal boundary conditions. Secular equations for the half-space in closed form and isolated mathematical conditions in completely separate terms are derived.

Recent development in science and technology requires that the high-performance electromechanical devices must have a higher sensitivity and larger storage capacity but

(C) 2019 Vietnam Academy of Science and Technology 
as maller size. Nano scale materials and structures have been introduced and developed ever since. For these materials, the conventional continuum elasticity theory fails to represent the constitutive relationships properly [5]. A non-local model based on Eringens theory of non-local continuum mechanics has been proposed for the effects of the size dependency in very small structures. Particularly, Eringen's nonlocal theory [6] has been extended to study the size dependent mechanical performances of the piezoelectric nanostructures by Ke et al. [7-9]. There are a few research works on the propagation of the guided elastic waves in nanoscale periodic piezoelectric structures. For examples, Chen et al. [10] studied the anti-plane transverse wave propagation in nanoscale periodic layered piezoelectric structures. Yan et al. [11,12] investigated the propagation of guided elastic waves in nanoscale layered periodic piezoelectric composites.

However, only few researchs [11-13] on wave propagation in nanoscale periodic layered piezoelectric structures have been reported in literature due to the complexity of the problem. In addition, many researches $[1,14]$ have been carried out on the propagation of Rayleigh waves in transversely isotropic piezoelastic materials solids. However, to the best of the authors knowledge, there are no researches analyzing the propagation of surface waves in transversely isotropic nonlocal piezoelastic half-space analytically available in the literature. Therefore, the main purpose of this paper is to study the effect of nonlocality on the propagation of Rayleigh waves in transversely isotropic piezoelastic materials. The dispersion equation for the propagation of Rayleigh waves is derived for the boundary is stress-free, maintained at charge-free condition.

\section{FORMULATION OF THE PROBLEM}

We consider homogeneous transversely isotropic, electrically conducting piezoelectric medium in the undeformed state at initial potential $\phi_{0}$. We assume that the medium is transversely isotropic in such a way that planes of isotropy are perpendicular to $x_{3}-$ axis. We take the origin of the coordinate system $\left(x_{1}, x_{2}, x_{3}\right)$ at any point on the plane surface and $x_{3}$-axis pointing vertically downward into the half-space. Thus the considering half-space is represented by $x_{3} \geq 0$. For two-dimensional problem in which the plane wave is in the plane $x_{1} x_{3}$ ), the strains are related to the displacement field $u_{1}, u_{3}$ and the electric potential $\phi$. The constitutive equations are given as [1,11]:

- Strain-displacement relations

$$
\varepsilon_{11}=u_{1,1}, \quad \varepsilon_{33}=u_{3,3}, \quad \varepsilon_{13}=\varepsilon_{31}=\frac{1}{2}\left(u_{1,3}+u_{3,1}\right),
$$

- Stress-strain and electric field relations

$$
\begin{aligned}
& \sigma_{11}=c_{11} \varepsilon_{11}+c_{13} \varepsilon_{33}-e_{31} E_{3}, \quad \sigma_{33}=c_{13} \varepsilon_{11}+c_{33} \varepsilon_{33}-e_{33} E_{3}, \\
& \sigma_{13}=\sigma_{31}=2 c_{44} \varepsilon_{13}-e_{15} E_{1}, \\
& D_{1}=2 e_{15} \varepsilon_{13}+\epsilon_{11} E_{1}, \quad D_{3}=e_{13} \varepsilon_{11}+e_{33} \varepsilon_{33}+\epsilon_{33} E_{3},
\end{aligned}
$$


where $E_{i}=-\phi_{, i}$ is the electric field and $D_{i}$ the electric displacement, $\rho$ the mass density, $\sigma_{i j}$ the stress tensor, $c_{i j}$ the elastic parameters tensor, $e_{i j}$ the piezoelectric moduli, $\epsilon_{i j}$ the electric permittivity $(i, j=1,3)$.

It is well known that in the classical piezoelectricity (CPE) theory, the stresses and the electric displacements at one point only dependend on the local strains and electric fields at the same point. But when the macro size reaches a few nanometers, the CPE continuum theory fails and we have usually to utilize other methods. The essence of the Eringen's nonlocal elasticity theory $[6,15]$ is that the stress at a point $x$ in a body depends not only on the strain at that point but also on the strain at all other points $x^{\prime}$ in the domain. Recently, Ke et al. $[7,8]$ extended the nonlocal elasticity theory to the piezoelectric nanostructuresthe nonlocal continuum theory of piezoelectricity (NLPE). Unlike the CPE continuum theory, the NLPE theory supposes that the stresses and the electrical displacements at one point should be affected by the strains and electrical fields at all points of the whole body. Thus the relationship between the CPE stress and electrical displacement components and the NLPE stress and electrical displacement components can be written as $[11,12]$

$$
t_{m n}=\left(1+\epsilon^{2} \nabla^{2}\right) \sigma_{m n}, \quad d_{m}=\left(1+\epsilon^{2} \nabla^{2}\right) D_{m},
$$

where $t_{m n}$ and $d_{m}$ are the NLPE stress and electrical displacement components, respectively; $\sigma_{m n}$ and $D_{m}$ are the traditional stress and electrical displacement components, respectively. Constant $\epsilon\left(=e_{0} a\right)$ is the nonlocal parameter $\left(e_{0}\right.$ is the nonlocal constant and $a$ is the internal characteristic length).

For the wave propagation considered in this paper, the body forces, electric charge are ignored. Using the relations (3), the motion equations, Gauss equation are simplified as $[11,12]$

$$
\begin{aligned}
& \sigma_{11,1}+\sigma_{13,3}=\left(1-\epsilon^{2} \nabla^{2}\right) \rho \ddot{u_{1}}, \quad \sigma_{13,1}+\sigma_{33,3}=\left(1-\epsilon^{2} \nabla^{2}\right) \rho \ddot{u_{3}}, \\
& D_{1,1}+D_{3,3}=0 .
\end{aligned}
$$

Substituiting (2) into (4) taking into account (1) we have

$$
\begin{aligned}
& c_{11} u_{1,11}+c_{44} u_{1,33}+\left(c_{13}+c_{44}\right) u_{3,13}+\left(e_{15}+e_{31}\right) \phi_{, 13}=\left(1-\epsilon^{2} \nabla^{2}\right) \rho \ddot{u}_{1}, \\
& \left(c_{13}+c_{44}\right) u_{1,13}+c_{44} u_{3,11}+c_{33} u_{3,33}+e_{15} \phi_{, 11}+e_{33} \phi_{, 33}=\left(1-\epsilon^{2} \nabla^{2}\right) \rho \ddot{u}_{3}, \\
& \left(e_{15}+e_{31}\right) u_{1,13}+e_{15} u_{3,11}+e_{33} u_{3,33}-\epsilon_{11} \phi_{, 11}-\epsilon_{33} \phi_{, 33}=0 .
\end{aligned}
$$

For the waves propagating in the plane $x_{3}=0$, we take the form of relevant components of displacement and the electric potential $\phi$ as $[1,16]$

$$
\left\{\begin{array}{l}
u_{1}=a_{1} e^{-\tilde{\xi} y} e^{i k\left(x_{1}-c t\right)} \\
u_{3}=a_{3} e^{-\xi y} e^{i k\left(x_{1}-c t\right)} \\
\phi=A_{1} e^{-\xi y} e^{i k\left(x_{1}-c t\right)}
\end{array} \quad \text { with } \quad y=k x_{3},\right.
$$

where $a_{1}, a_{3}, A_{1}$ are polarization vectors, $k$ is wavenumber, $c$ is speed of wave propagation, $\xi$ is a complex coefficient whose imaginary part should be positive corresponding to the decay condition in the half-space $x_{3}>0$.

Substituting the expressions for displacement and electric potential from (6) into (5), we obtain the three homogeneous equations in three unknowns $a_{1}, a_{3}, A_{1}$. For a nontrivial 
solution of these equations, we must have $\operatorname{det}(\mathbf{M})=0$, in which

$$
\mathbf{M}=\left[\begin{array}{ccc}
c_{11}-c_{44} \xi^{2}-\rho c^{2}-\rho c^{2} k^{2} \epsilon^{2}\left(1-\xi^{2}\right) & \left(c_{13}+c_{44}\right) i \xi & \left(e_{15}+e_{31}\right) i \xi \\
\left(c_{13}+c_{44}\right) i \xi & c_{44}-c_{33} \xi^{2}-\rho c^{2}-\rho c^{2} k^{2} \epsilon^{2}\left(1-\xi^{2}\right) & e_{15}-e_{33} \xi^{2} \\
\left(e_{15}+e_{31}\right) i \xi & e_{15}-e_{33} \xi^{2} & \epsilon_{33} \xi^{2}-\epsilon_{11}
\end{array}\right] .
$$

This is the characteristics equation and it has the form

$$
h_{6} p^{6}+h_{4} p^{4}+h_{2} p^{2}+h_{0}=0,
$$

where $p=i \xi$ and the coefficients $h_{i},(i=0,2,4,6)$ are given in the Appendix.

Eq. (8) is a cubic polynomial in $p^{2}$. We order $p_{n}, n=1,2, \ldots, 6$, in such a way that $p_{1}, p_{2}, p_{3}$ correspond to waves traveling in the positive $x_{3}$ direction, and $p_{4}, p_{5}, p_{6}$ correspond to the ones traveling in the negative $x_{3}$, respectively. Since, we are interested in surface waves only so it is essential that motion is confined to free surface $x_{3}=0$ of the half-space so that the characteristic roots $p_{i}^{2}$ must satisfy the radiation condition $\operatorname{Im}\left(p_{i}\right) \geq 0$. Then the general solution for displacements and electric potential are written as [1]

$$
\left\{\begin{array}{l}
u_{1}=\sum_{j=1}^{3} a_{1 j} e^{p_{j} y} e^{i k\left(x_{1}-c t\right)} \\
u_{3}=\sum_{j=1}^{3} a_{3 j} e^{p_{j} y} e^{i k\left(x_{1}-c t\right)} \\
\phi=\sum_{j=1}^{3} A_{1 j} e^{p_{j} y} e^{i k\left(x_{1}-c t\right)}
\end{array}\right.
$$

where $a_{1 j}, a_{3 j}$ and $A_{1 j}$ are the amplitudes of the displacements and the electric potentials, respectively.

Remark: For the propagation of plane waves with phase velocity $c$ in the direction making an angle $\theta$ with the vertical axis, a surface wave of this form is expressed by

$$
\left\{\begin{array}{l}
u_{1}=a_{1} e^{i k\left(p_{1} x_{1}+p_{3} x_{3}-c t\right)} \\
u_{3}=a_{3} e^{i k\left(p_{1} x_{1}+p_{3} x_{3}-c t\right)} \\
\phi=A_{1} e^{i k\left(p_{1} x_{1}+p_{3} x_{3}-c t\right)}
\end{array}\right.
$$

where $p_{1}=\sin \theta, p_{3}=\cos \theta$ are components of propagation unit vector. Substituting (10) into (5) and obtain a matrix similar to the matrix $\mathbf{M}$ in Eq. (7). By letting the determinant of this matrix equal zero, we have a quadratic equation in $c^{2}$. Therefore, we obtain two real roots $c_{j}(j=1,2)$ corresponding the speeds of plane waves propagating in the medium.

\section{BOUNDARY CONDITIONS AND DISPERSION EQUATIONS}

In this section, the Rayleigh wave equation for transversely isotropic nonlocal piezoelastic half-space can be derived using the boundary conditions at the surface of the halfspace. In the present study, boundary conditions appropriate for particle motion in the $x_{1} x_{3}$ plane are considered at the plane surface $x_{3}=0$. This surface is considered to be 
stress-free (mechanical conditions), which requires the normal stress $\sigma_{33}$ as well as the tangential stress $\sigma_{13}$ to vanish at the surface $x_{3}=0$. That means

$$
\sigma_{13}=\sigma_{33}=0 .
$$

Another condition is required to represent that the surface of half-space is maintained at charge free condition (open circuit-surface), namely

$$
D_{3}=0 .
$$

Substituting (9) into the boundary conditions (11), (12) and taking into account (2), we have a system of linear equations

$$
\left\{\begin{array}{l}
\sum_{j=1}^{3}\left[c_{44}\left(a_{3 j}+a_{1 j} p_{j}\right)+e_{15} A_{1 j}\right]=0, \\
\sum_{j=1}^{3}\left[c_{13} a_{1 j}+c_{33} a_{3 j} p_{j}+e_{33} A_{1 j} p_{j}\right]=0, \\
\sum_{j=1}^{3}\left[e_{13} a_{1 j}+e_{33} a_{3 j} p_{j}-\epsilon_{33} A_{1 j} p_{j}\right]=0 .
\end{array}\right.
$$

For each $p_{j}(j=1,2,3)$, the three corresponding unknowns $a_{1 j}, a_{3 j}, A_{1 j}(7)$ are in a relationship given by matrix $\mathbf{M}$ and we can express them as $a_{1 j}=\alpha_{j} A_{1 j}, a_{3 j}=\beta_{j} A_{1 j}$ where

$$
\begin{aligned}
\alpha_{j}= & \frac{\left(c_{13}+c_{44}\right) p_{j}\left(e_{15}+e_{33} p_{j}^{2}\right)-\left(c_{44}+c_{33} p_{j}^{2}-\rho c^{2}-\rho c^{2} k^{2} \epsilon^{2}\left(1+p_{j}^{2}\right)\right)\left(e_{15}+e_{31}\right) p_{j}}{\delta_{j}}, \\
\beta_{j}= & \frac{\left(c_{13}+c_{44}\right) p_{j}^{2}\left(e_{15}+e_{31}\right)-\left(c_{11}+c_{44} p_{j}^{2}-\rho c^{2}-\rho c^{2} k^{2} \epsilon^{2}\left(1+p_{j}^{2}\right)\right)\left(e_{15}+e_{33} p_{j}^{2}\right)}{\delta_{j}}, \\
\delta_{j}= & {\left[c_{11}+c_{44} p_{j}^{2}-\rho c^{2}-\rho c^{2} k^{2} \epsilon^{2}\left(1+p_{j}^{2}\right)\right]\left[c_{44}+c_{33} p_{j}^{2}-\rho c^{2}-\rho c^{2} k^{2} \epsilon^{2}\left(1+p_{j}^{2}\right)\right] } \\
& -\left(c_{13}+c_{44}\right)^{2} p_{j}^{2}, \quad j=1,2,3 .
\end{aligned}
$$

Then we obtain a system of linear equations in amplitudes $A_{11}, A_{12}, A_{13}$ only and it is in the form

$$
\left\{\begin{array}{l}
\left(c_{44} \beta_{1}+c_{44} \alpha_{1} p_{1}+e_{15}\right) A_{11}+\left(c_{44} \beta_{2}+c_{44} \alpha_{2} p_{2}+e_{15}\right) A_{12}+\left(c_{44} \beta_{3}+c_{44} \alpha_{3} p_{3}+e_{15}\right) A_{13}=0, \\
\left(c_{13} \alpha_{1}+c_{33} \beta_{1} p_{1}+e_{33} p_{1}\right) A_{11}+\left(c_{13} \alpha_{2}+c_{33} \beta_{2} p_{2}+e_{33} p_{2}\right) A_{12}+\left(c_{13} \alpha_{3}+c_{33} \beta_{3} p_{3}+e_{33} p_{3}\right) A_{13}=0 \\
\left(e_{31} \alpha_{1}+e_{33} \beta_{1} p_{1}-\epsilon_{33} p_{1}\right) A_{11}+\left(e_{31} \alpha_{2}+e_{33} \beta_{2} p_{2}-\epsilon_{33} p_{2}\right) A_{12}+\left(e_{31} \alpha_{3}+e_{33} \beta_{3} p_{3}-\epsilon_{33} p_{3}\right) A_{13}=0 .
\end{array}\right.
$$

The dispersion equation of Rayleigh waves is obtained from $\operatorname{det}(\mathbf{C O})=0$ where matrix $\mathrm{CO}$ is the matrix of coefficients of the system of equation above as

$$
\left[\begin{array}{ccc}
c_{44} \beta_{1}+c_{44} \alpha_{1} p_{1}+e_{15} & c_{44} \beta_{2}+c_{44} \alpha_{2} p_{2}+e_{15} & c_{44} \beta_{3}+c_{44} \alpha_{3} p_{3}+e_{15} \\
c_{13} \alpha_{1}+c_{33} \beta_{1} p_{1}+e_{33} p_{1} & c_{13} \alpha_{2}+c_{33} \beta_{2} p_{2}+e_{33} p_{2} & c_{13} \alpha_{3}+c_{33} \beta_{3} p_{3}+e_{33} p_{3} \\
e_{31} \alpha_{1}+e_{33} \beta_{1} p_{1}-\epsilon_{33} p_{1} & e_{31} \alpha_{2}+e_{33} \beta_{2} p_{2}-\epsilon_{33} p_{2} & e_{31} \alpha_{3}+e_{33} \beta_{3} p_{3}-\epsilon_{33} p_{3}
\end{array}\right] .
$$


This dispersion equation is in implicit form and it shows the relation between the phase velocity $c$ and the wave number $k$ of the Rayleigh waves and the parameters of the medium.

\section{NUMERICAL RESULTS AND DISCUSSION}

In order to illustrate theoretical results obtained in the preceding sections, the material chosen for the numerical calculations is CdSe (6 mm class) of hexagonal symmetry, which is transversely isotropic material. The physical data for a single crystal of CdSe material is given below $[1,14]$

$$
\begin{aligned}
& c_{11}=7.41 \times 10^{10} \mathrm{Nm}^{-2}, c_{13}=3.93 \times 10^{10} \mathrm{Nm}^{-2}, c_{33}=8.36 \times 10^{10} \mathrm{Nm}^{-2}, \\
& c_{44}=1.32 \times 10^{10} \mathrm{Nm}^{-2}, \rho=5504 \mathrm{kgm}^{-3}, e_{15}=-0.138 \mathrm{Cm}^{-2}, e_{31}=-0.16 \mathrm{Cm}^{-2}, \\
& e_{33}=0.347 \mathrm{Cm}^{-2}, \epsilon_{11}=8.26 \times 10^{-11} \mathrm{C}^{2} \mathrm{~N}^{-1} \mathrm{~m}^{-2}, \epsilon_{33}=9.03 \times 10^{-11} \mathrm{C}^{2} \mathrm{~N}^{-1} \mathrm{~m}^{-2}, \\
& e_{0}=0.39, a=0.421 \times 10^{-9} \mathrm{~m}, \epsilon=e_{0} a .
\end{aligned}
$$

Denote $e p=k^{2} \epsilon^{2}$ the dimensionless frequency where $k$ is the wavenumber. This is an important parameter that provides us the information of the wave-length of Rayleigh waves comparing to the nonlocal parameter of the medium.

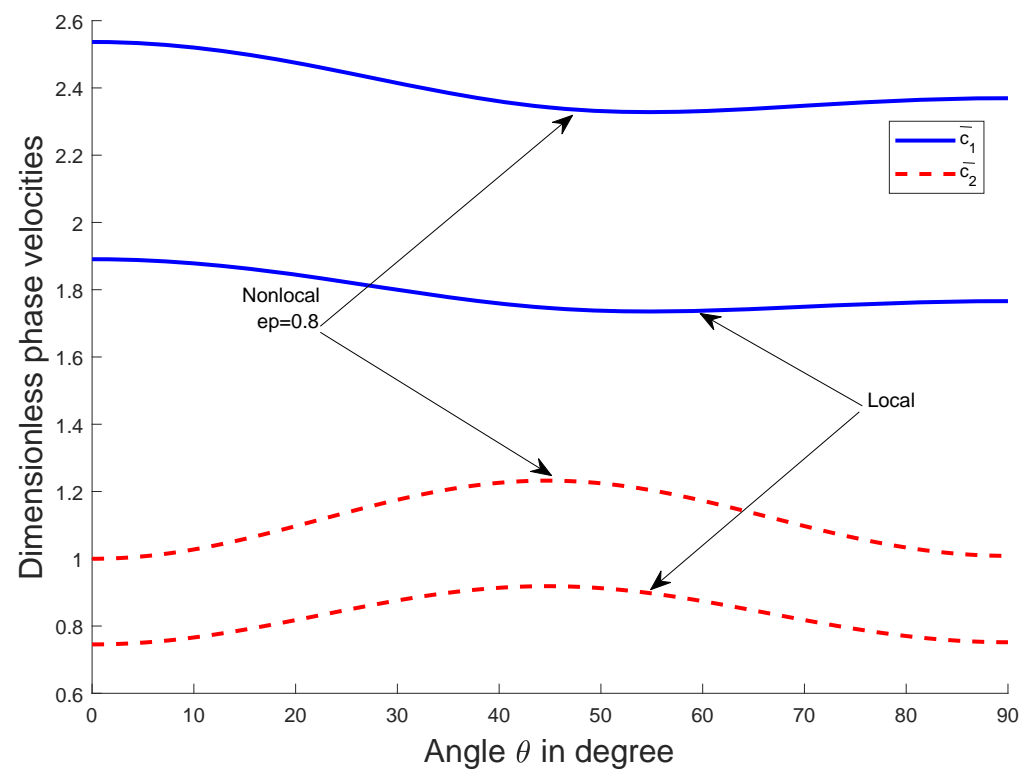

Fig. 1. Dimensionless velocities of plane waves $\bar{c}_{1}$ and $\bar{c}_{2}$ depending on the angle directions $\theta$ for local and nonlocal case

First, we evaluate the effect of nonlocality to the speed of plane waves. Fig. 1 shows the dimentionless speed of plane waves $c / b_{S}$, where $b_{S}=\sqrt{c_{44} / \rho}$ is the speed of SHtype wave, depending on the direction of propagation $\theta$ (angle between the direction 
of propagation and vertical axis) in the piezoelectric medium for two case of local theory and nonlocal theory with the nonlocal parameter given by ep $=0.8$. It can be seen that the phase velocities $c_{1}, c_{2}$ in the nonlocal theory case are greater the ones in the local theory case. It can be concluded that the nonlocality has significant effect on the velocities of propagation of plane waves.

Next, the variations of the phase velocities with dimensionless parameter ep for $\theta=$ $\pi / 3$ are depicted in Fig. 2. Generally, this figure shows that the phase velocities $c_{1}, c_{2}$ are decreasing when $e p$ is increasing. When $e p<1$ these velocities decrease rapidly decrease while e $p>1$ they are quite stable.

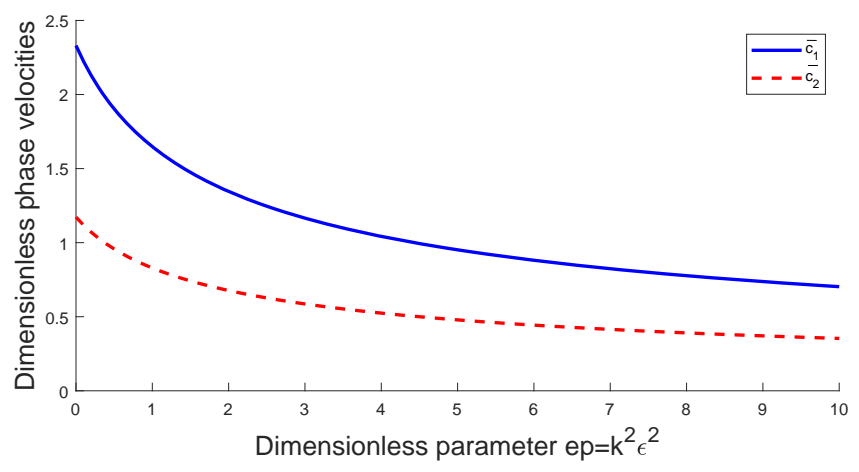

Fig. 2. The comparison of variations of the phase velocities with dimensionless parameter $e p=k^{2} \epsilon^{2}$ for $\theta=\pi / 3$

Finally, the dimensionless speed of Rayleigh wave $x=X / c_{44}$ with $X=\rho c^{2}$ depends upon the dimensionless parameter ep is illustrated by Fig. 3 for the boundary condition of open circuit surface (maintained charge free). The speed of Rayleigh wave is decreasing when the parameter $e p$ is increasing.

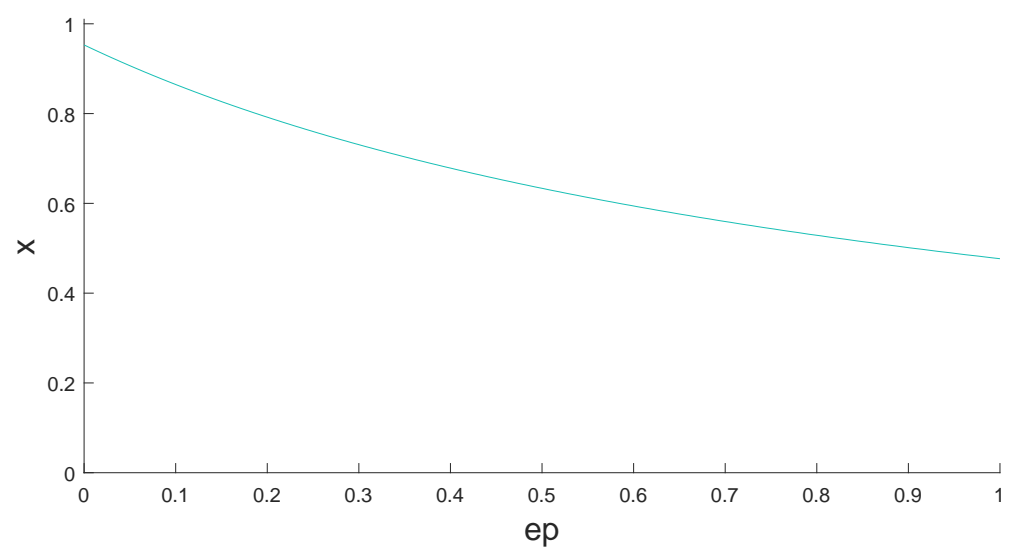

Fig. 3. Effect of parameter ep on the speed of Rayleigh wave $x$ for the open circuit surface 


\section{CONCLUSIONS}

In the present work, we have studied the propagation of Rayleigh waves in transversely isotropic piezoelastic nonlocal materials. Some important features are drawn below:

(i) Under certain type of specific boundary condition: the surface $x_{3}=0$ is considered to be stress-free and maintained at charge-free condition, the dispersion equation of the Rayleigh waves is given. It is numerically concluded that the nonlocality has significant effect on the speed of Rayleigh wave.

(ii) Phase velocities of plane waves are computed numerically and their variation on the incident angle $\theta$, dimensionless frequency parameter ep, are presented graphically. The effect the nonlocality on the velocities of plane waves are also expressed through numerical example and the effect is also significant.

\section{ACKNOWLEDGMENT}

The work was supported by the Vietnam National Foundation for Science and Technology Development (NAFOSTED) under Grant 107.02-2019.06.

\section{REFERENCES}

[1] J. N. Sharma, M. Pal, and D. Chand. Propagation characteristics of Rayleigh waves in transversely isotropic piezothermoelastic materials. Journal of Sound and Vibration, 284, (1-2), (2005), pp. 227-248. https://doi.org/10.1016/j.jsv.2004.06.036.

[2] J. Yang. An introduction to the theory of piezoelectricity. Springer, (2005).

[3] L. P. Zinchuk and A. N. Podlipenets. Dispersion equations for Rayleigh waves in a piezoelectric periodically layered structure. Journal of Mathematical Sciences, 103, (3), (2001), pp. 398403. https://doi.org/10.1023/A:1011382816558.

[4] A. K. Vashishth and V. Gupta. Wave propagation in transversely isotropic porous piezoelectric materials. International Journal of Solids and Structures, 46, (20), (2009), pp. 3620-3632. https://doi.org/10.1016/j.ijsolstr.2009.06.011.

[5] G. Z. Voyiadjis. Handbook of nonlocal continuum mechanics for materials and structures. Springer Nature Switzerland AG, (2018).

[6] A. C. Eringen and D. G. B. Edelen. On nonlocal elasticity. International Journal of Engineering Science, 10, (3), (1972), pp. 233-248. https://doi.org/10.1016/0020-7225(72)90039-0.

[7] L.-L. Ke and Y.-S. Wang. Thermoelectric-mechanical vibration of piezoelectric nanobeams based on the nonlocal theory. Smart Materials and Structures, 21, (2), (2012). https://doi.org/10.1088/0964-1726/21/2/025018.

[8] L.-L. Ke, Y.-S. Wang, and Z.-D. Wang. Nonlinear vibration of the piezoelectric nanobeams based on the nonlocal theory. Composite Structures, 94, (6), (2012), pp. 2038-2047. https://doi.org/10.1016/j.compstruct.2012.01.023.

[9] L.-H. Ma, L.-L. Ke, Y.-Z. Wang, and Y.-S. Wang. Wave propagation analysis of piezoelectric nanoplates based on the nonlocal theory. International Journal of Structural Stability and Dynamics, 18, (04), (2018). https://doi.org/10.1142/S0219455418500608.

[10] A.-L. Chen, D.-J. Yan, Y.-S. Wang, and C. Zhang. Anti-plane transverse waves propagation in nanoscale periodic layered piezoelectric structures. Ultrasonics, 65, (2016), pp. 154-164. https://doi.org/10.1016/j.ultras.2015.10.006. 
[11] D.-J. Yan, A.-L. Chen, Y.-S. Wang, C. Zhang, and M. Golub. Propagation of guided elastic waves in nanoscale layered periodic piezoelectric composites. European Journal of MechanicsA/Solids, 66, (2017), pp. 158-167. https://doi.org/10.1016/j.euromechsol.2017.07.003.

[12] D.-J. Yan, A.-L. Chen, Y.-S. Wang, C. Zhang, and M. Golub. In-plane elastic wave propagation in nanoscale periodic layered piezoelectric structures. International Journal of Mechanical Sciences, 142, (2018), pp. 276-288. https://doi.org/10.1016/j.ijmecsci.2018.04.054.

[13] F.-M. Li and Y.-S. Wang. Study on localization of plane elastic waves in disordered periodic 2-2 piezoelectric composite structures. Journal of Sound and Vibration, 296, (3), (2006), pp. 554566. https://doi.org/10.1016/j.jsv.2006.01.057.

[14] J. N. Sharma and V. Walia. Further investigations on Rayleigh waves in piezothermoelastic materials. Journal of Sound and Vibration, 301, (1-2), (2007), pp. 189-206. https://doi.org/10.1016/j.jsv.2006.09.018.

[15] A. C. Eringen. On differential equations of nonlocal elasticity and solutions of screw dislocation and surface waves. Journal of Applied Physics, 54, (9), (1983), pp. 4703-4710. https://doi.org/10.1063/1.332803.

[16] P. C. Vinh, V. T. N. Anh, D. X. Tung, and N. T. Kieu. Homogenization of very rough interfaces for the micropolar elasticity theory. Applied Mathematical Modelling, 54, (2018), pp. 467-482. https://doi.org/10.1016/j.apm.2017.09.039.

\section{APPENDIX}

The coefficients of characteristic equation

$$
\begin{aligned}
& h_{4}=-b_{9} b_{3}^{2}+2 b_{3} b_{4} b_{7}-b_{5} b_{4}^{2}-b_{2} b_{7}^{2}-2 b_{1} b_{8} b_{7}+b_{1} b_{5} b_{10}+b_{1} b_{6} b_{9}+b_{2} b_{5} b_{9}, \\
& h_{2}=-b_{3}^{2} b_{10}+2 b_{3} b_{4} b_{8}-b_{6} b_{4}^{2}-b_{1} b_{8}^{2}-2 b_{2} b_{7} b_{8}+b_{1} b_{6} b_{10}+b_{2} b_{5} b_{10}+b_{2} b_{6} b_{9}, \\
& h_{0}=b_{2} b_{6} b_{10}-b_{2} b_{8}^{2}, h_{6}=b_{1} b_{5} b_{9}-b_{1} b_{7}^{2}, b_{1}=c_{44}-X k^{2} \epsilon^{2}, b_{2}=c_{11}-X-X k^{2} \epsilon^{2}, \\
& b_{3}=c_{13}+c_{44}, b_{4}=e_{15}+e_{31}, b_{5}=c_{33}-X k^{2} \epsilon^{2}, b_{6}=c_{44}-X-X k^{2} \epsilon^{2}, \\
& b_{7}=e_{33}, b_{8}=e_{15}, b_{9}=-\epsilon_{33}, b_{10}=-\epsilon_{11}, X=\rho c^{2} .
\end{aligned}
$$

